\title{
CD59 is overexpressed in human lung cancer and regulates apoptosis of human lung cancer cells
}

\author{
BAIJUN LI ${ }^{*}$, HUI LIN ${ }^{2 *}$, JIAN FAN $^{1}$, JIAO LAN ${ }^{2}$, YONGLONG ZHONG $^{1}$, \\ YONG YANG $^{1}$, HUI LI ${ }^{1}$ and ZHIWEI WANG ${ }^{1}$ \\ ${ }^{1}$ Renmin Hospital of Wuhan University, Wuhan 430060; ${ }^{2}$ The People's Hospital of Guangxi \\ Zhuang Autonomous Region, Nanning 530000, P.R. China
}

Received March 21, 2013; Accepted May 9, 2013

DOI: 10.3892/ijo.2013.2007

\begin{abstract}
CD59, belonging to membrane complement regulatory proteins (mCRPs), inhibits the cytolytic activity of complement and is overexpressed in many types of solid cancers. The aim of the present study was to detect the expression of CD59 in non-small cell lung cancer (NSCLC) and to investigate the relationship between decreased CD59 expression and tumorigenesis of NSCLC by transfecting recombinant retrovirus encoding shRNA targeting human CD59 into the human NSCLC cell line NCI-H157. CD59 expression in NSCLC was detected by immunocytochemistry (IHC). In the human NSCLC cell line NCI-H157, CD59 mRNA and protein expression suppressed with lentivirus-mediated RNAi was confirmed by using RT-PCR and western blotting, respectively. The proliferation and apoptosis of NCI-H157 cells was measured by using MTT assay and FACS. The resistance to complement cracking ability was detected by LDH assay. Caspase-3 expression in cells was assessed by IHC. Bcl-2 and Fas protein was determined by western blotting both in vitro and in vivo. CD59 is overexpressed in human NLCLC cancer. In NCI-H157 cells, lentivirus-mediated RNAi significantly reduced both CD59 mRNA and protein expression, which resulted in suppressing cell proliferation and increasing cell apoptosis. When incubated with fresh normal human serum $(8 \%, v / v)$ for $1 \mathrm{~h}$ at $37^{\circ} \mathrm{C}$, the cell viability was decreased and cell apoptosis was increased in siCD59-infected NCI-H157 cells compared to siCD59-C-infected cells. Reduced CD59 expression led to increased expression of caspase -3 and Fas and decreased expression of Bcl-2. Furthermore, the nude mouse tumor graft weight was significantly decreased and survival rate was significantly increased in the siCD59 group. CD59 is overexpressed in human NLCLC. CD59 silencing in NSCLC cancer cells via retrovirus-mediated RNAi can
\end{abstract}

Correspondence to: Professor Zhiwei Wang, Renmin Hospital of Wuhan University, Wuhan 430060, P.R. China

E-mail:wangzhiwp@sina.com

*Contributed equally

Key words: CD59, apoptosis, membrane attack complex, non-small cell lung cancer enhance complement-mediated cell apoptosis, inhibiting the growth of NSCLC. CD59 may serve as a potential target for gene therapy in NSCLC.

\section{Introduction}

Lung cancer is the most common cancer worldwide and the leading cause of cancer-related mortality (1). Non-small cell lung cancer (NSCLC) accounts for $\sim 80 \%$ of all lung cancer cases and has a 5-year overall survival rate of $<15 \%(2,3)$. Approximately $40 \%$ of patients diagnosed with NSCLC have unresectable stage III disease or inoperable disease (4). Therefore, additional efforts are needed to understand and identify molecular targets for gene therapy $(5,6)$.

The cluster of differentiation 59 (CD59), also called protectin, is a type of complement regulated proteins (CRPs). CD59 inhibits the complement cytolytic activity by binding to C8 and C9, which blocks the assembly of the membrane attack complex (MAC) (7). CD59 is overexpressed in most solid malignancies and presents at low levels in normal tissues. CD59 overexpression may assist malignant cells to escape immunologic surveillance and complement-mediated cytolysis, limiting the effect of complement-fixing monoclonal antibodies (7-10). Immune escape of tumor cells is a primary cause of failed immunotherapy. Blocking of CD59 function on the surface of tumor cells might allow effective complement-mediated clearance of tumor cells that improve the effect of complementactivating antitumor antibodies $(11,12)$. Therefore, CD59 is a promising therapeutic target for antitumor gene therapy.

RNA interference (RNAi) is an economical, fast and highly efficient technique for silencing gene expression (13-15). Recently, siRNA-encoding plasmids delivered by virus has been rapidly developed and widely applied in mammalian cells $(16,17)$. In this study, we examined the expression of CD59 in NSCLC and constructed a CD59 small interfering RNA (siRNA) lentiviral vector. We assessed its effect on the proliferation and apoptosis of lung cancer cells and further characterized the functional role of CD59 during lung cancer tumorigenesis.

\section{Materials and methods}

Expression of CD59 in tissue specimens by immunohistochemistry (IHC). Twenty primary NSCLC specimen and 
corresponding surgical margin specimens were obtained from the People's Hospital of Guangxi Zhuang Autonomous Region. A mouse polyclonal antibody for CD59 was obtained from Santa Cruz Biotechnology (SC-133171) to assess the expression of the CD59 protein. Briefly, paraffin-embedded tissue sections $(5-\mu \mathrm{m}$ thick) were deparaffinized with xylene and then dehydrated in sequential diluted ethanol before rinsing in PBS. Sections were heated for $10 \mathrm{~min}$ in $0.01 \mathrm{M}$ citrate buffer (pH 6.0) twice to unmask the antigens. Endogenous peroxidase activity was then blocked with $3 \%$ hydrogen peroxide for $20 \mathrm{~min}$ at room temperature. Before being incubated with CD59 antibody (dilution, $1 / 100$ in $0.01 \mathrm{M} \mathrm{PBS}$ ) at $4^{\circ} \mathrm{C}$ overnight, sections were incubated with $5 \%$ normal goat serum in $1 \%$ BSA in PBS for $30 \mathrm{~min}$ to block non-specific IgG binding. A biotinylated goat antimouse $\mathrm{IgG}$ was used for further incubation and a strepavidin-biotin complex system (SABC) with diaminobenzidene as chromogen was used for color development. The sections were weakly counterstained with hematoxylin before mounting and then examined under a light microscope. PBS $(0.01 \mathrm{M})$ was used to replace primary antibody to serve as negative staining controls. Immunohistochemical staining was evaluated by two independent pathologists.

Construction of small interfering RNA targeted CD59 expression vector, production of lentivirus and transfection into NCI-H 157 cells. The pSUPER vector was digested by BglII and HindIII restriction enzyme and annealed oligos, siCD59: 5'-GATCCCCGCGTGTCTCATTACCAAAGttcaagagaCTT TGGTAATGAGACACGTTTTA-3. siCD59-C: 5'-AGCTTA AAAAGCGTGTCTCATTACCAAagtctcttgAACTTTGGTA ATGAGACACGCGGG-3' were ligated with this vector. The recombinants were identified by PCR, restriction endonuclease analyses and DNA sequencing, respectively.

A packaging cell line Phoenix A and human non-small lung cancer cell line NCI-H157 cells (18) were cultured in DMEM and RPMI-1640 supplemented with 10\% FBS (Gibco, Carlsbad, CA, USA) and penicillin/streptomycin, incubating in a humidified incubator $\left(37^{\circ} \mathrm{C}, 5 \% \mathrm{CO}_{2}\right)$. For retroviral production, Phoenix A cells were transfected with this recombinant using Lipofectamine 2000 (Invitrogen, Carlsbad, CA, USA) according to the manufacturer's instructions. Culture supernatants were collected after $48 \mathrm{~h}$. NCI-H157 cells were infected with siCD59 and siCD59-C in the presence of $8 \mathrm{~g} / \mathrm{ml}$ polybrene, follow by clonal selection with $\mathrm{G} 418(400 \mathrm{mg} / \mathrm{l})$ to generate stable clones.

Detection of the expression of CD59 mRNA by real-time quantitative PCR (RT-qPCR) test. Total RNA from different groups of NCI-H157 cells was isolated using TRIzol reagent (Invitrogen). Two microgram of total RNA was reverse-transcribed in a $20-\mu 1$ reaction using reverse-transcription system (Promega, Madison, WI, USA). Primers were designed based on sequences of human CD59 and $\beta$-actin. The forward primer of CD59 was 3'-ACACCATTGCTGGGGACCTC-5' and the reverse primer was 3'-GCTGAATCTTAAAGTCAGGCAA AGG-5'. The forward primer of $\beta$-actin was 3'-CACACCCGC CACCAGTTCGC-5' and the reverse primer was 3'-AGCACAG GGTGCTCCTCAGGG-5'. The amplified sequence was 356 and $332 \mathrm{bp}$, respectively. Thermo cycling was carried out as follows: CD59; $94^{\circ} \mathrm{C}$ for $5 \mathrm{~min}$, then 40 cycles of $94^{\circ} \mathrm{C}$ for
$45 \mathrm{sec}, 52^{\circ} \mathrm{C}$ for $45 \mathrm{sec}$ and $72^{\circ} \mathrm{C}$ for $45 \mathrm{sec}$, followed by $72^{\circ} \mathrm{C}$ for $7 \mathrm{~min}$ or $\beta$-actin $94^{\circ} \mathrm{C}$ for $5 \mathrm{~min}$, then 40 cycles of $94^{\circ} \mathrm{C}$ for $40 \mathrm{sec}, 58^{\circ} \mathrm{C}$ for $35 \mathrm{sec}$ and $72^{\circ} \mathrm{C}$ for $45 \mathrm{sec}$, followed by $72^{\circ} \mathrm{C}$ for $7 \mathrm{~min}$. PCR products were quantified by using Quantity One 6.4.0 (Bio-Rad, Hercules, CA, USA) Software. CD59 levels were normalized to $\beta$-actin.

Determination of CD59 protein expression by western blotting. Different groups of NCI-H157 cells were harvested at the indicated time-points, washed twice with cold phosphatebuffered saline (PBS), lysed in fresh cell lysis buffer for $2 \mathrm{~h}$ on ice and centrifuged at $12,000 \mathrm{~g}$ for $15 \mathrm{~min}$ at $4^{\circ} \mathrm{C}$ to remove insoluble materials. Protein concentrations were determined by BCA assay. Protein $(20 \mu \mathrm{g})$ was separated by using 8 and $5 \%$ sodium dodecyl sulfate-polyacrylamide gel electrophoresis and transferred to PVDF membranes. The membrane was incubated with anti-CD59 (1:500) and anti- $\beta$-actin (1:200) antibodies (Santa Cruz Biotechnology, Inc., Santa Cruz, CA, USA), respectively, followed by incubation with horseradish peroxidase-conjugated goat anti-mouse secondary antibody. Western blots were developed by chemiluminescence and quantified using Quantity One 6.4.0 (Bio-Rad) software. CD59 levels were normalized to $\beta$-actin.

Determination of the NSCLC cell proliferation by MTT assay. Different groups of NCI-H157 cells were cultured for 3 days in 96-well plates and then incubated in $5 \%$ MTT at $37^{\circ} \mathrm{C}$ for $4 \mathrm{~h}$. DMSO (100 $\mu \mathrm{l} /$ well) was added and the light absorption value at $490 \mathrm{~nm}$ was measured.

Determination of the NSCLC cell apoptosis by flow cytometry. Different groups of NCI-H157 cells were seeded in 6-well culture plates. Each group contained five culture flasks. After $24 \mathrm{~h}$, the cells were harvested and washed in cold PBS. Annexin V and PI staining were carried out using the Annexin V-FITC Apoptosis Detection kit (BD Biosciences, USA), according to the manufacturer's protocol. After a 20-min incubation in the dark at room temperature, the cells were immediately analyzed by FACSCalibur Flow Cytometry (BD Biosciences).

Determination of the NSCLC cell resistance to complement cracking ability by $\mathrm{LDH}$ release test. Different groups of NCI-H157 cells were treated with complement inactivated $8 \%$ fresh normal human serum (NHS) for $60 \mathrm{~min}$ at $37^{\circ} \mathrm{C}$. Triton X-100 (0.1\%) in RPMI-1640 was used as the $100 \%$ lysis control and RPMI-1640 alone was used for the $0 \%$ lysis control. Following incubation, $40 \mu \mathrm{l}$ of sample supernatant was taken for LDH assay. To each well $100 \mu \mathrm{l}$ solution $\mathrm{C}$ was added. Just before analysis, $10 \mu \mathrm{l}$ solution $\mathrm{B}$ was added. The absorbance at $440 \mathrm{~nm}$ was calculated, reflecting the activity of LDH present and the following equation applied: LDH leakage rate.

Detection of the expression of caspase-3 in cells by immunohistochemistry (IHC). Different groups of NCI-H157 cells $\left(2 \times 10^{4} \mathrm{ml}\right)$ were seeded onto glass coverslips for $48 \mathrm{~h}$. After incubation with fresh NHS for $6 \mathrm{~h}$, the cover slips were washed thrice with PBS, 2\% PBS-paraformaldehyde solution was added for $15 \mathrm{~min}$ at room temperature and $0.4 \%$ Triton X-100 was added at room temperature for $15 \mathrm{~min}$. After washing thrice with PBS, the cells were treated with $30 \% \mathrm{H}_{2} \mathrm{O}_{2}$. The 
A

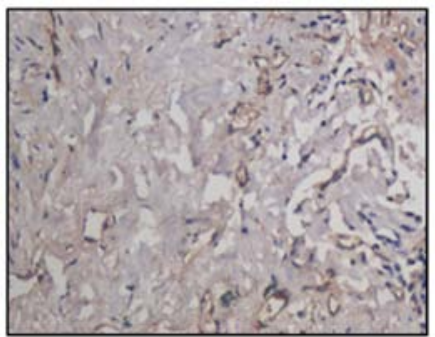

Precancerous tissue
B

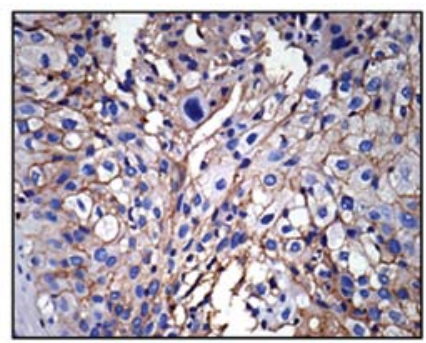

Cancer tissue
Figure 1. Expression of CD59 in non-small cell lung cancer. (A) Expression of CD59 in the surrounding tissue. (B) Expression of CD59 in the non-small cell lung cancer.

caspase-3 protein expression levels were measured by IHC staining. In brief, cells were incubated in blocking buffer at room temperature for $20 \mathrm{~min}$, followed by the rabbit antihuman caspase- 3 antibody (1:500 Abcam, Cambridge, MA, USA) at $4^{\circ} \mathrm{C}$ overnight followed by an additional washing step (3X) with PBS. Secondary antibody labeled with HRP (mouse anti-rabbit) was added and incubated at $37^{\circ} \mathrm{C}$ for $1 \mathrm{~h}$ followed by washing (3X) with PBS. SABC was added at $37^{\circ} \mathrm{C}$ for $20 \mathrm{~min}$ and $\mathrm{DAB}$ at room temperature for 5-30 min and the results were observed under a light microscope. Positive cells were stained brownish yellow. Caspase-3 protien levels were determined by positive index $=$ positive percentage $(\%) \mathrm{x}$ mean optical density x 100 .

Determination of Bcl-2 and Fas protein by western blotting. Total protein extraction of different groups of NCI-H157 was performed, as described above. Twenty microgram $(20 \mu \mathrm{g})$ was separated using 8 and 5\% sodium dodecyl sulfatepolyacrylamide gel electrophoresis and transferred to PVDF membranes. The membrane was incubated with anti-bcl-2 $(1: 500)$, anti-Fas $(1: 10,000)$ and anti- $\beta$-actin $(1: 200)$ antibodies (Santa Cruz), followed by incubation with horseradish peroxidase-conjugated goat anti-mouse secondary antibody. Western blots were developed by chemiluminescence and bands were quantified by using Quantity One 6.4.0 (Bio-Rad) software. CD59 levels were normalized to $\beta$-actin.

Xenograft studies. Athymic male nude mice (5- to 6-week-old) were purchased from Laboratory Animal Centre of Guangxi
Medical University and housed under pathogen-free conditions. All animal experiments were reviewed and approved by the Institutional Animal Care and Use Committee of the Guangxi Medical University. Thirty mice were randomly divided into three groups of 10 each: control group, siCD59 groups and siCD59-ctr groups. Each mouse of every group was subcutaneously respectively injected with $2 \times 10^{5}$ luciferaseexpressing Luc-NCI-H157 cell, siCD59 and siCD59-ctr cells (Invitrogen) into the right flank. Tumor growth/regression was monitored every 5 day by in vivo imaging after intraperitoneal injection of firefly luciferin $(150 \mathrm{mg} / \mathrm{kg})$ to the mice using an non-invasive imaging system (Roper, USA). Each mouse cohort was also monitored for 60 days to determine the tumor volume and the survival rate.

Determination of CD59 mRNA and CD59, Bcl-2 and Fas protein expression in vivo. Mice were sacrificed at the end of the experiments by $\mathrm{CO}_{2}$ asphyxiation. Tumors were excised and immediately snap-frozen in liquid nitrogen. Total RNA and protein extraction of tumor tissue were frozen in liquid nitrogen. CD59 mRNA expression was determined by RT-PCR and CD59, Bcl-2, Fas protein was determined by western blotting.

Statistical analysis. Data were analyzed using GraphPad Prism 5.0 statistical software. Quantitative data were expressed as the means \pm standard deviation. Significant difference was determined by $\mathrm{P}$-values $<0.05$.

\section{Results}

Expression of CD59 in lung cancer. CD59 expression was significantly higher in tissues from non-small cell lung carcinoma (NSCLC) than in preneoplastic tissue (67.6 vs. $4.3 \%$, $\mathrm{P}<0.05$ ) (Fig. 1).

CD59-siRNA efficiently suppressed CD59 expression. Recombinant retroviruses siCD59 and siCD59-control (siCD59-C) both contain a green fluorescent protein (GFP) reporter gene, which allowed for measuring infection efficiency in NCI-H157 cells. Forty-eight hours post-infection, the infection efficiency was $70 \%$ in both siCD59 and siCD59-C infected cells (Fig. 2). CD59 mRNA and protein levels were decreased significantly in siCD59 infected cells compared to siCD59-C infected cells (Fig. 3).

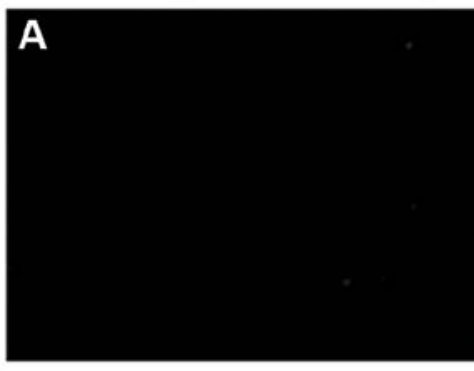

Non-infected cells

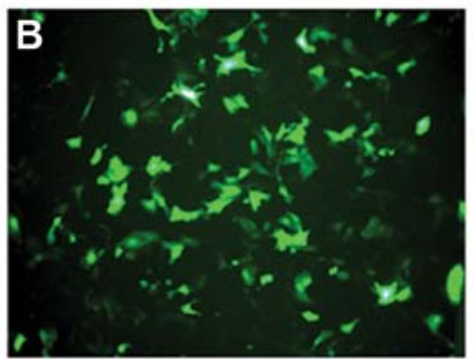

siCD59 infected cells

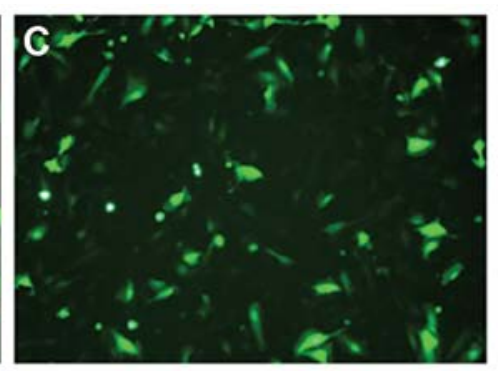

Ctr-siCD59 infected cells

Figure 2. Fluorescence microscope images of NCI-H157 cells $48 \mathrm{~h}$ after infection. (A) Luc-NCI-H157 cells without infection. (B) Luc-NCI-H157 cells infected by siCD59. (C) Luc-NCI-H157 cells infected by siCD59-C. 
A

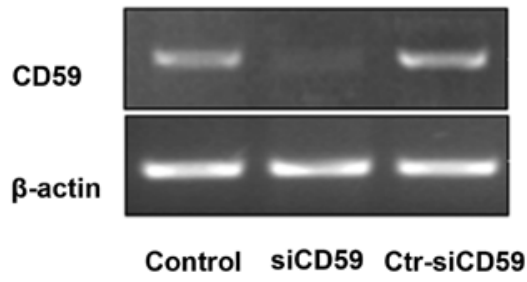

B

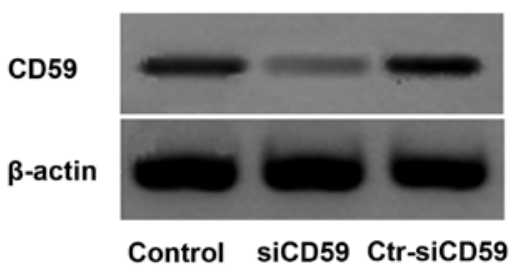

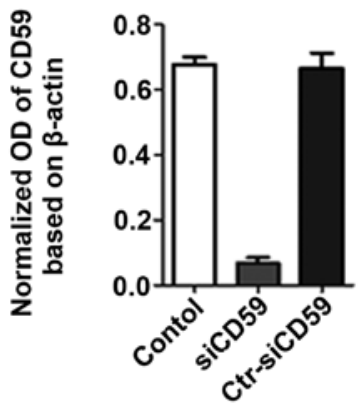

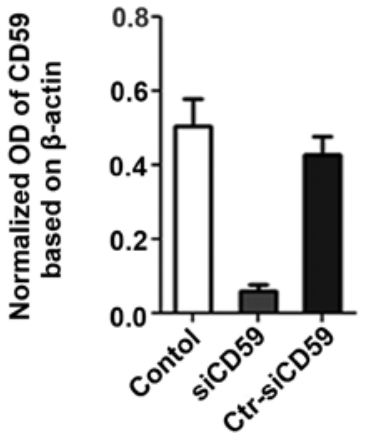

Figure 3. (A) Expression of CD59 mRNA in Luc-NCI-H157 cells, Luc-NCI-H157 (siCD59) and Luc-NCI-H157 (siCD59-ctr) cells. siCD59 significantly decrease the CD59 mRNA expression and the control siRNA did not affect the CD59 expression. (B) Expression of CD59 protein in Luc-NCI-H157 cells, Luc-NCI-H157 (siCD59) and Luc-NCI-H157 (siCD59-ctr) cells. siCD59 significantly decrease the CD59 protein expression, and the control siRNA did not affect the CD59 expression.

A

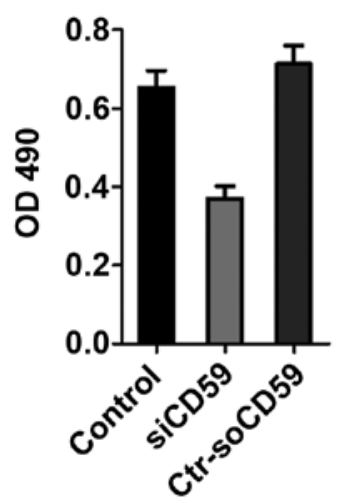

B

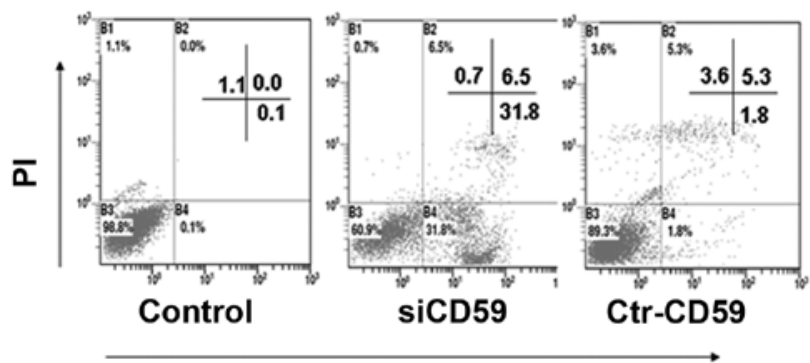

Annexin V

Figure 4. (A) Effect of CD59-siRNA on lung cancer cell proliferation. The proliferation of siCD59 transfected cells decreased significantly compared with the Luc-NCI-H157 and siCD59-ctr transfected cells. (B) Effect of CD59-siRNA on lung cancer cell apoptosis. The apoptosis of siCD59 transfected cells increased significantly compared with the Luc-NCI-H157 and siCD59-ctr transfected cells.

Effect of CD59-siRNA on lung cancer cell proliferation and apoptosis. To determine the effect of CD59 knockdown on the growth of NCI-H157 cells, an MTT assay was performed on NCI-H157 cells, siCD59 and siCD59-C transfected NCI-H157 cells. SiCD59-transfected NCI-H157 cells displayed a significant decrease in grow th rate compared to siCD59-C-transfected cells and NCI-H157 cells (Fig. 4A). To determine the effects of CD59 knockdown on cell apoptosis, flow cytometry were used. Knockdown of CD59 increased apoptosis of NCI-H157 cells compared to NCI-H157 cells and siCD59-ctr cells (31.8\% $\mathrm{P}>0.1 \%, \mathrm{P}>0.05 ; 31.8 \% \mathrm{P}>0.18 \%, \mathrm{P}>0.05$ ) (Fig. 4B)

CD59-siRNA reduced cell viability and increased cell damage when treated with complement. The viability and
Table I. LDH test.

\begin{tabular}{lc}
\hline Groups & LDH release rate \\
\hline Cells only & $9.6 \pm 1.3$ \\
Cells + normal serum & $17.4 \pm 3.7$ \\
siCD59 + normal serum & $63.5 \pm 5.3$ \\
Ctr-siCD59 + normal serum & $16.3 \pm 4.1$ \\
\hline
\end{tabular}

cellular DNA damage of siCD59-NCI-H157 transfected cells was also reduced and increased, respectively, compared to the siCD59-C cells and NCI-H157 cells (Table I). 


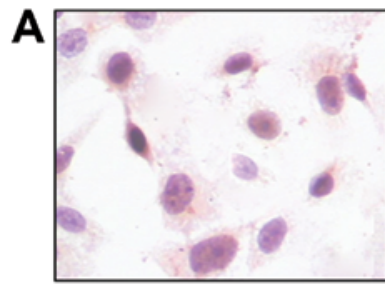

$\mathrm{NCl}-\mathrm{H} 157$ cells

B
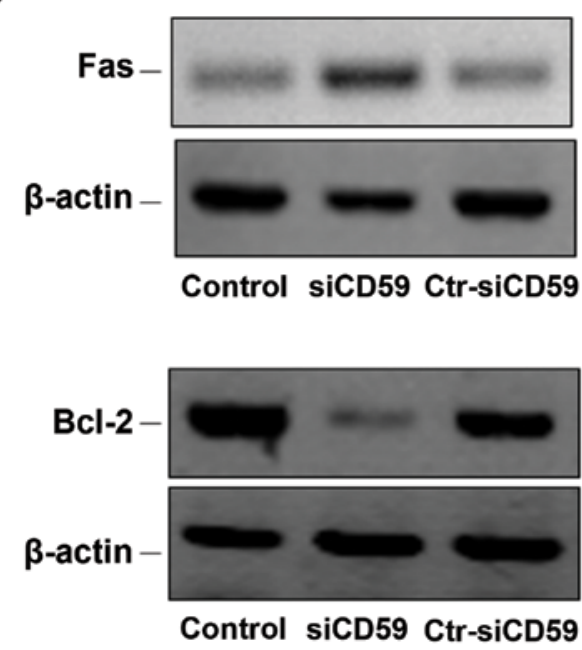

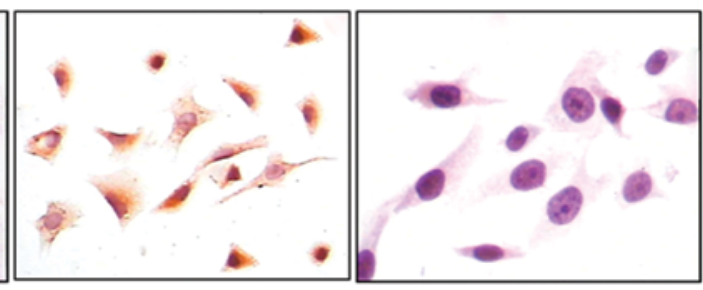

siCD59

Ctr-siCD59

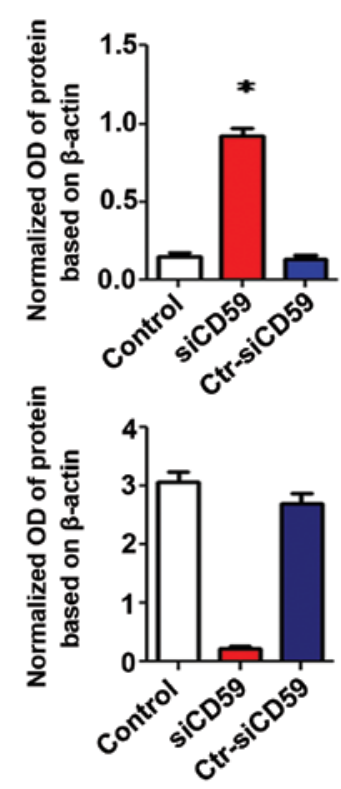

Figure 5. (A) The expression of apoptosis-associated protein caspase-3 in NCI-H157 cells, NCI-H157 (siCD59) and NCI-H157 (siCD59-ctr) cells. siCD59 significantly increased the caspase-3 protein expression, but the control siRNA did not affect the caspase- 3 expression. (B) The expression of apoptosis-associated protein Fas and Bcl-2 in NCI-H157 cells, NCI-H157 (siCD59) and NCI-H157 (siCD59-ctr) cells. siCD59 significantly increased the Fas and decreased Bcl-2 protein expression, whereas the control siRNA did not affect the Fas or Bcl-2 expression. These results also confirm the caspase-3 result.

CD59-siRNA affects expression of caspase-3, Fas and Bcl-2. Shi et al (6) demonstrated that apoptosis could modulate the expression of CD59. IHC and western blot analysis showed increased caspase-3 (Fig. 5A) and Fas (Fig. 5B) levels while Bcl-2 (Fig. 5B) levels were decreased in siCD59-transfected NCI- H157 cells and NCI-H157 cells. These results indicate that CD59 regulates apoptosis in non-small cell lung cancer cells.

Knockdown of CD59 suppresses lung cancer cell growth in vivo. To test whether loss of CD59 can suppress lung cancer progression, we performed a xenograft study whereby different groups of NCI-H157 cells were subcutaneously injected into mice. Tumors were allowed to reach a size such that $\sim 10^{8}$ photons $/ \mathrm{sec} / \mathrm{cm}^{2}$ were emitted following luciferin processing. Subsequently, luciferase signals in tumors of mice were detected in 10, 15 and 20 days after postinjection. Compared to s-CD59-C tumors and NCI-H157 cells, significant decrease in tumor burden was observed upon CD59 knockdown (Fig. 6A and B). The survival rate of tumor-bearing mice at 60 days was 70,0 and $0 \%$ in siCD59-transfected tumors group, siCD59-C tumors group and NCI- H157 tumors group (Fig. 6C).

In vivo expression of CD59 mRNA and CD59, Bcl-2 and Fas protein. Total RNA and protein were extracted from tumor tissues to determine CD59 levels. Robust decrease in CD59 levels at both the RNA and protein levels were observed in siCD59 cells compared to siCD59-C cells and NCI- H157 cells (Fig. 7A). Furthermore, Fas and Bcl-2 expression was significantly increased and decreased, respectively, in siCD59 cells compared to siCD59-C and NCI- H157 cells (Fig. 7B).

\section{Discussion}

Human CD59 is an $18-20 \mathrm{kDa}$ protein anchored through glycanphosphatidylinositol (GPI) to the cell membrane (19). CD59 belongs to the members of Ly6 superfamily (20). The functions of CD59 protein are mainly involved in the following three aspects. First, CD59 functions as an inhibitor of the C5b-9 membrane attack complex (MAC) of human complement (21). Second, CD59 acts as the second signal stimulant, inducing the activation of $\mathrm{T}$ lymphocytes and taking part in the regulatory course of immunoreactions (22). Third, CD59 is the ligand of CD2 that can conglutinate with CD59. CD59CD2 complex activates $\mathrm{T}$ cells and then guides adhesion of $\mathrm{T}$ and $\mathrm{T}$ cells or $\mathrm{T}$ and other tissue cells and further regulates the growth of tissue cells (23).

Previous studies have shown that CD59 is highly expressed in many types of tumors, including breast cancer (24-27), colorectal cancer $(26,28,29)$, ovarian cancer $(30)$, malignant gliomas (31), malignant lymphomas (32), prostate cancer (33) and pancreatic cancer (34) and it directly or indirectly 
A

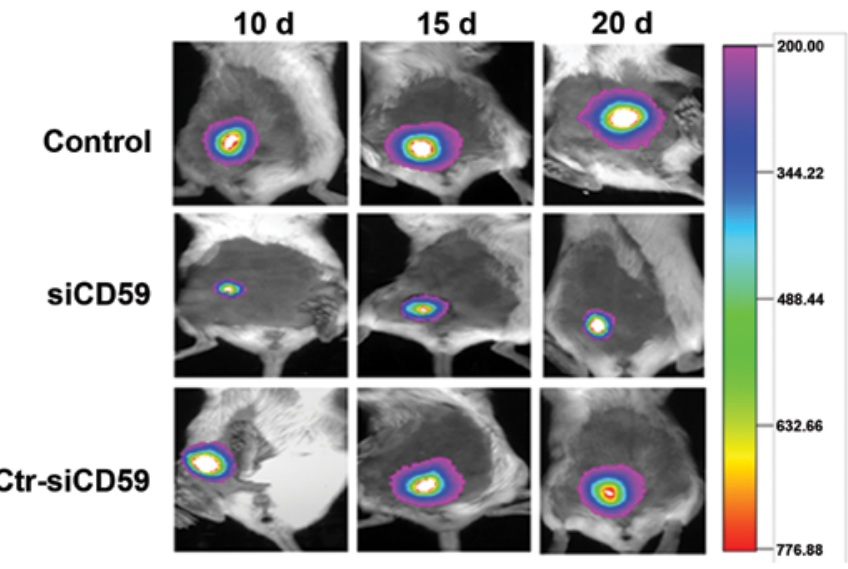

B
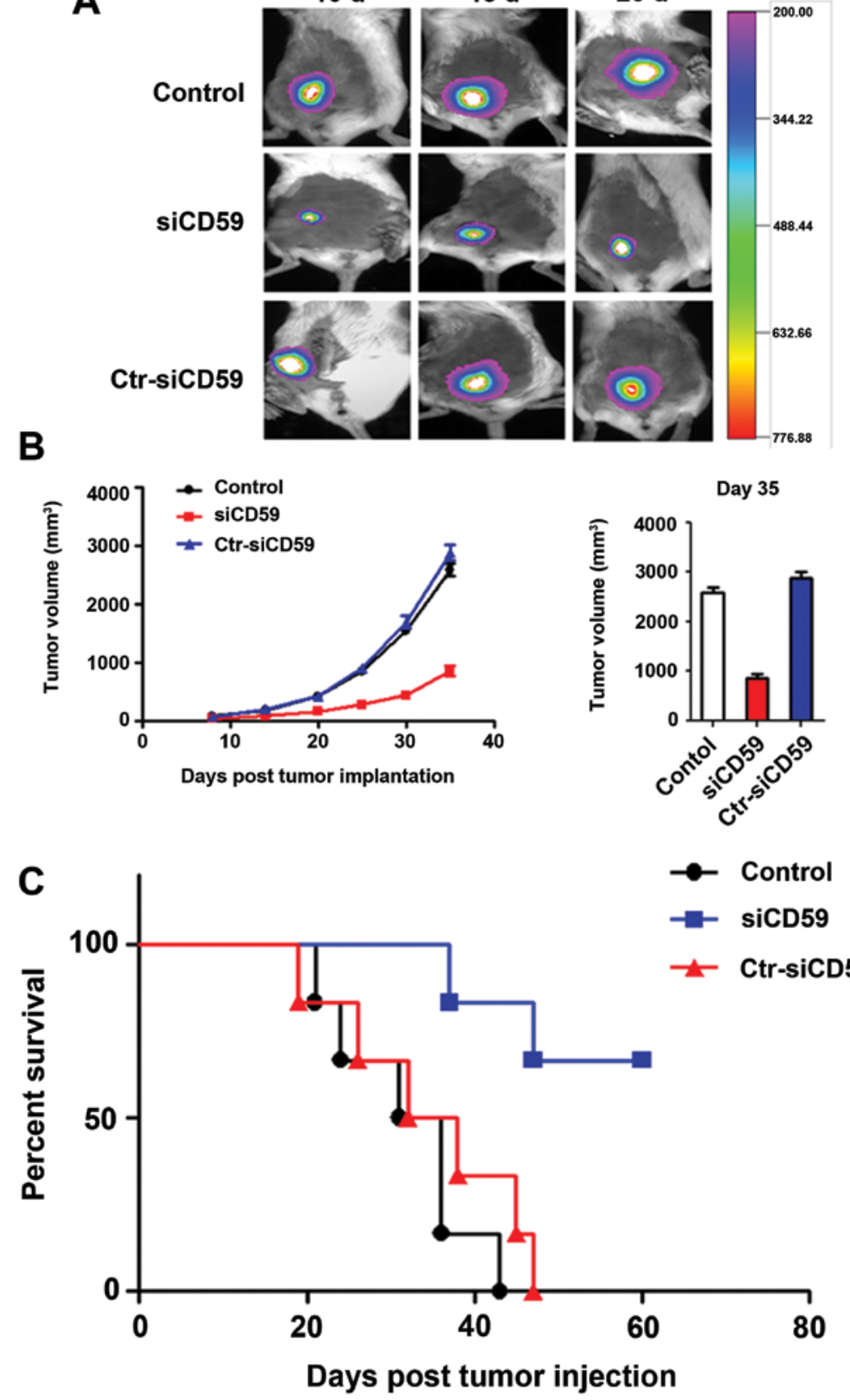

Figure 6. (A) Luciferase activity in tumors. Luciferase signals in tumors was decreased in siCD59 transfected mice in comparison to controls groups and siCD59-ctr treated groups. (B) Tumor size. Seven days after tumor injection, tumor growth and tumor size were observed and results showed that tumor growth was more rapid in the control group and siCD59-ctr transfected group than siCD59 transfected group. (C) Survival rate of tumor-bearing mice. Sixty days after tumor injection, all of the mice had died in Luc-NCI-H157 group and siCD59-ctr transfected group while in siCD59 transfected group only 3 had died.

participates in carcinogenesis and tumorigenesis. Our studies focused on the role of CD59 during tumorigenesis of NSCLC cells (NCI-H157) upon silencing CD59.

In the present study, we first detected high expression of CD59 in tissues of patients with lung cancer (Fig. 1). CD59 expression in non-small cell lung cancer tissues is much higher than in the surrounding tissue based on IHC. This suggests that CD59 might be a new biomarker for lung cancer progression.

RNA interference has emerged as a genetic tool for silencing gene expression. Because siRNA can be integrated into the host genome, long-term gene silencing can be achieved of siRNAs $(17,35)$. Retroviral infection of siCD59 to NCI-H157 cells was able to significantly decrease CD59 mRNA and protein levels compared to siCD59-C cells (Fig. 3). Knockdown of CD59 decreased cell viability and increased cell damage and apoptosis of lung cancer cells (Fig. 4). Furthermore, CD59 knockdown also reduced tumor growth in vivo (Fig. 6).

Loss of CD59 can induce complement-mediated cytolysis and lead to apoptosis of tumor cells (5). Complement-mediated cytolysis can also assist in apoptosis by inducing phagocytosis $(36,37)$. MAC-triggered cell death can occur through a caspase-dependent pathway, specifically via caspase-3. Animal models of renal disease also implicate that MAC can trigger apoptosis (38-40). Korty et al demonstrated that CD59 can also increase calcium flux to increase cytoplasmic 


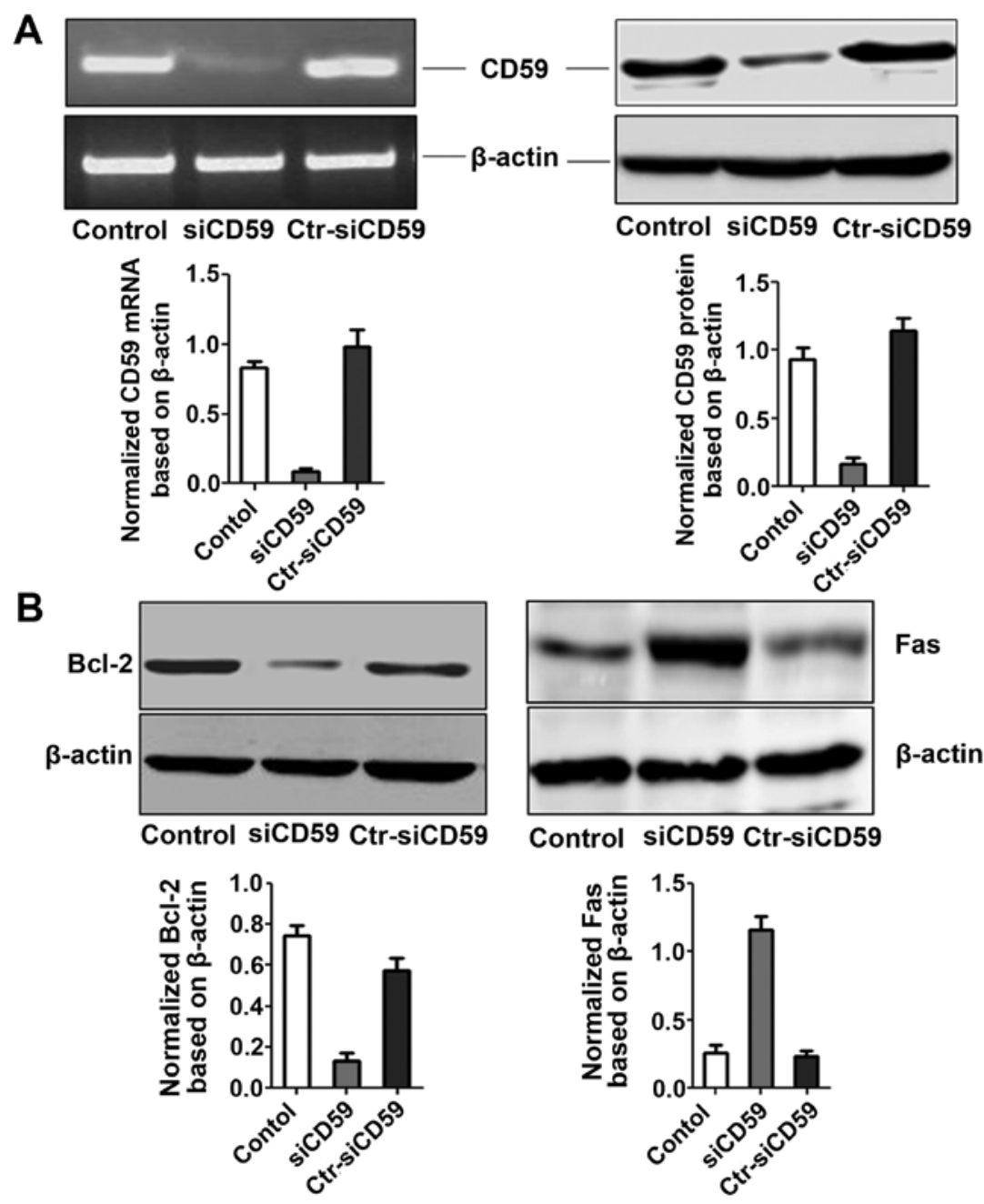

Figure 7. (A) CD59 expression in tumor tissues. The CD59 expression of mRNA and protein is dramatically less in siCD59 transfected group. (B) Fas and Bcl-2 expression in tumor tissues. The Fas is significantly increased and the Bcl-2 is dramatically reduced in siCD59 transfected group.

calcium levels which induces mitochondrial DNA damage and cytochrome $c(41)$. Fas expression also initiates apoptosis under certain conditions and Fas antigen and ICAM-1 molecule can have a synergistic effect (42). Bcl-2 has also emerged as a proto-oncogene that blocks programmed cell death independently of promoting cell division (43). Bcl-2 can inhibit the synthesis and activation of caspase- 3 to inhibit apoptosis. Moreover, Bcl-2 can also be degraded by caspase-3 a specific enzyme, which activates apoptosis. Apoptosis can inhibit tumorigenesis by removing unwanted and damaged cells (52).

Cell apoptosis typically occurs during tumor development and regression. Therefore, an important mechanism to prevent tumorigenesis is the induction of cell apoptosis that takes place continuously in many tissues to remove unwanted, damaged or aberrant cells. In this study, we investigated the effects of CD59 silencing to understand the biochemical mechanism underlying CD59 inactivation-induced apoptosis. We also studied the role of CD59 in regulating the growth and apoptosis of NCI-H157 cells. We showed that CD59 knockdown significantly decreased the growth and increased apoptosis of NCI-H157 cells compared to siCD59-C cells (Fig. 4), consistent with previous observations $(44,45)$. CD59-induced apoptosis might be mediated by MAC. Further studies are needed to understand this mechanism.

NCI-H157 cells treated by human complement (NHS, 8\%, v/v) were used to assess MAC-mediated cytolysis. We showed that CD59 knockdown significantly inhibited the viability of NCI-H157 cells (Table I), decreased Bcl-2 expression and increased caspase-3 and Fas expression (Fig. 5). Caspases are executioners of apoptosis and regulate Fas/TNF-R1, mitochondria dysfunction and TNF-related apoptosis-inducing ligand (TRAIL). Caspase-3 activation was observed upon Fas/ TNF-R1 treatment (Fig. 5A). Overall, the present data suggest that the loss of CD59 induces caspase-dependent apoptosis in cultured cells.

To investigate the inhibitory role of siCD59 in vivo, siCD59 infected cells were injected into athymic nude mice. Knockdown of CD59 significantly decreased tumor weight and growth and increased the survival rate of mice compared to siCD59-C cells and NCI-H157 cells (Fig. 6), which suggests that silencing CD59 gene expression could markedly inhibit the growth of cancer in vivo. The silencing of CD59 expression in tumor tissue was confirmed by RT-PCR and western blotting (Fig. 7A), which showed significantly less CD59 expression in siCD59 group than in siCD59-C group, or the NCI-H157 cell 
group. Decreased tumor growth could be attributed to apoptosis, as indicated by Fas and Bcl-2 expression in these tumor tissues (Fig. 7B). The present data suggest that the CD59 loss induces caspase-dependent apoptosis in vitro and in vivo.

Overall, our study illustrates that CD59 is increased in human lung cancer and loss or inactivation of CD59 can lead to apoptosis of NCI-H157 lung cancer cells. This may be mediated by inducing Fas expression on the surface of NCI-H157 cells, leading to apoptosis through caspase-3 activation and complement-induced cytolysis. This might provide insight into new treatment for lung cancer patients by inhibiting CD59 expression.

In conclusion, the present study demonstrates that CD59 is overexpressed in human lung cancer and retroviral-mediated RNAi delivery is an efficient system for CD59 gene silencing of human lung cancer. Suppression of CD59 expression enhanced complement-mediated cytolysis of lung cancer cells, which may be mediated by induction of Fas expression on the surface of NCI-H157 cells, leading to apoptosis through caspase-3 activation, CD59 may serve as a candidate targeting gene in gene therapy for human carcinomas such as lung, ovarian, prostate carcinoma and cervical carcinoma.

\section{Acknowledgements}

This study was supported by Grants from the Scientific Research and Technological Development Projects of Guangxi, China (no. 0816004-8).

\section{References}

1. Parkin DM, Bray F, Ferlay J and Pisani P: Global cancer statistics, 2002. CA Cancer J Clin 55: 74-108, 2005.

2. Fidias P and Novello S: Strategies for prolonged therapy in patients with advanced non-small-cell lung cancer. J Clin Oncol 28: 5116-5123, 2010.

3. Fuld AD, Dragnev KH and Rigas JR: Pemetrexed in advanced non-small-cell lung cancer. Expert Opin Pharmacother 11: 1387-1402, 2010

4. Whitehurst AW, Bodemann BO, Cardenas J, et al: Synthetic lethal screen identification of chemosensitizer loci in cancer cells. Nature 446: 815-819, 2007.

5. Li B, Chu X, Gao M and Xu Y: The effects of CD59 gene as a target gene on breast cancer cells. Cell Immunol 272: 61-70, 2011

6. Shi XX, Zhang B, Zang JL, Wang GY and Gao MH: CD59 silencing via retrovirus-mediated RNA interference enhanced complement-mediated cell damage in ovary cancer. Cell Mol Immunol 6: 61-66, 2009.

7. Fonsatti E, Altomonte M, Coral S, et al: Emerging role of protectin (CD59) in humoral immunotherapy of solid malignancies. Clin Ter 151: 187-193, 2000.

8. Chen S, Caragine T, Cheung NK and Tomlinson S: CD59 expressed on a tumor cell surface modulates decay-accelerating factor expression and enhances tumor growth in a rat model of human neuroblastoma. Cancer Res 60: 3013-3018, 2000.

9. Wickham SE, Hotze EM, Farrand AJ, et al: Mapping the intermedilysin-human CD59 receptor interface reveals a deep correspondence with the binding site on CD59 for complement binding proteins C8alpha and C9. J Biol Chem 286: 20952-20962, 2011.

10. Gelderman KA, Tomlinson S, Ross GD and Gorter A: Complement function in $\mathrm{mAb}$-mediated cancer immunotherapy. Trends Immunol 25: 158-164, 2004.

11. Fishelson Z, Donin N, Zell S, Schultz S and Kirschfink M: Obstacles to cancer immunotherapy: expression of membrane complement regulatory proteins (mCRPs) in tumors. Mol Immunol 40: 109-123, 2003

12. Fonsatti E, Di Giacomo AM and Maio M: Optimizing complement-activating antibody-based cancer immunotherapy: a feasible strategy? J Transl Med 2: 21, 2004.
13. Sioud M: Promises and challenges in developing RNAi as a research tool and therapy. Methods Mol Biol 703: 173-187, 2011.

14. Ashihara E: RNA interference for cancer therapies. Gan To Kagaku Ryoho 37: 2033-2041, 2010.

15. Ashihara E, Kawata E and Maekawa T: Future prospect of RNA interference for cancer therapies. Curr Drug Targets 11: 345-360, 2010.

16. Brummelkamp TR, Bernards R and Agami R: A system for stable expression of short interfering RNAs in mammalian cells. Science 296: 550-553, 2002.

17. Sui G, Soohoo C, Affar el B, Gay F, Shi Y, Forrester WC and Shi Y: A DNA vector-based RNAi technology to suppress gene expression in mammalian cells. Proc Natl Acad Sci USA 99: 5515-5520, 2002

18. Shafer SH and Williams CL: Non-small and small cell lung carcinoma cell lines exhibit cell type-specific sensitivity to edelfosine-induced cell death and different cell line-specific responses to edelfosine treatment. Int J Oncol 23: 389-400, 2003.

19. Geis N, Zell S, Rutz R, et al: Inhibition of membrane complement inhibitor expression (CD46, CD55, CD59) by siRNA sensitizes tumor cells to complement attack in vitro. Curr Cancer Drug Targets 10: 922-931, 2010.

20. Fleming TJ, O'HUigin C and Malek TR: Characterization of two novel Ly-6 genes. Protein sequence and potential structural similarity to alpha-bungarotoxin and other neurotoxins. J Immunol 150: 5379-5390, 1993.

21. Sugita Y, Nakano Y, Oda E, Noda K, Tobe T, Miura NH and Tomita M: Determination of carboxyl-terminal residue and disulfide bonds of MACIF (CD59), a glycosyl-phosphatidylinositol-anchored membrane protein. J Biochem 114: 473-477, 1993.

22. Treon SP, Shima Y, Grossbard ML, Preffer FI, Belch AR, Pilarski LM and Anderson KC: Treatment of multiple myeloma by antibody mediated immunotherapy and induction of myeloma selective antigens. Ann Oncol 11 (Suppl 1): 107-111, 2000.

23. Zaltzman AB, Van den Berg CW, Muzykantov VR and Morgan BP: Enhanced complement susceptibility of avidinbiotin-treated human erythrocytes is a consequence of neutralization of the complement regulators CD59 and decay accelerating factor. Biochem J 307: 651-656, 1995.

24. Hakulinen $\mathrm{J}$ and Meri S: Expression and function of the complement membrane attack complex inhibitor protectin (CD59) on human breast cancer cells. Lab Invest 71: 820-827, 1994.

25. Madjd Z, Pinder SE, Paish C, Ellis IO, Carmichael $\mathrm{J}$ and Durrant LG: Loss of CD59 expression in breast tumours correlates with poor survival. J Pathol 200: 633-639, 2003.

26. Thorsteinsson L, O'Dowd GM, Harrington PM and Johnson PM: The complement regulatory proteins CD46 and CD59, but not CD55, are highly expressed by glandular epithelium of human breast and colorectal tumour tissues. APMIS 106: 869-878, 1998.

27. Macor P, Mezzanzanica D, Cossetti C, Alberti P, Figini M, Canevari $\mathrm{S}$ and Tedesco F: Complement activated by chimeric anti-folate receptor antibodies is an efficient effector system to control ovarian carcinoma. Cancer Res 66: 3876-3883, 2006.

28. Koretz K, Brüderlein S, Henne C and Moller P: Expression of CD59, a complement regulator protein and a second ligand of the CD2 molecule, and CD46 in normal and neoplastic colorectal epithelium. Br J Cancer 68: 926-931, 1993.

29. Hosch SB, Scheunemann P, Lüth M, et al: Expression of 17-1A antigen and complement resistance factors CD55 and CD59 on liver metastasis in colorectal cancer. J Gastrointest Surg 5: 673-679, 2001.

30. Bjørge L, Hakulinen J, Wahlström T, Matre R and Meri S: Complement-regulatory proteins in ovarian malignancies. Int $\mathrm{J}$ Cancer 70: 14-25, 1997.

31. Mäenpää A, Junnikkala S, Hakulinen J, Timonen T and Meri S: Expression of complement membrane regulators membrane cofactor protein (CD46), decay accelerating factor (CD55), and protectin (CD59) in human malignant gliomas. Am J Pathol 148: 1139-1152, 1996.

32. Treon SP, Mitsiades C, Mitsiades N, Young G, Doss D, Schlossman R and Anderson KC: Tumor cell expression of CD59 is associated with resistance to $\mathrm{CD} 20$ serotherapy in patients with B-cell malignancies. J Immunother 24: 263-271, 2001.

33. Jarvis GA, Li J, Hakulinen J, Brady KA, Nordling S, Dahiya R and Meri S: Expression and function of the complement membrane attack complex inhibitor protectin (CD59) in human prostate cancer. Int J Cancer 71: 1049-1055, 1997. 
34. Crnogorac-Jurcevic T, Efthimiou E, Nielsen T, et al: Expression profiling of microdissected pancreatic adenocarcinomas. Oncogene 21: 4587-4594, 2002.

35. Liu CM, Liu DP, Dong WJ and Liang CC: Retrovirus vectormediated stable gene silencing in human cell. Biochem Biophys Res Commun 313: 716-720, 2004.

36. Riedemann NC, Guo RF, Laudes IJ, et al: C5a receptor and thymocyte apoptosis in sepsis. FASEB J 16: 887-888, 2002.

37. Guo RF, Huber-Lang M, Wang X, et al: Protective effects of anti-C5a in sepsis-induced thymocyte apoptosis. J Clin Invest 106: 1271-1280, 2000.

38. Niculescu T, Weerth S, Soane L, et al: Effects of membrane attack complex of complement on apoptosis in experimental autoimmune encephalomyelitis. Ann NY Acad Sci 1010: 530-533, 2003

39. Nauta AJ, Daha MR, Tijsma O, van de Water B, Tedesco F and Roos A: The membrane attack complex of complement induces caspase activation and apoptosis. Eur J Immunol 32: 783-792, 2002.

40. Hughes J, Nangaku M, Alpers CE, Shankland SJ, Couser WG and Johnson RJ: C5b-9 membrane attack complex mediates endothelial cell apoptosis in experimental glomerulonephritis. Am J Physiol Renal Physiol 278: F747-F7757, 2000.
41. Korty PE, Brando C and Shevach EM: CD59 functions as a signal-transducing molecule for human $\mathrm{T}$ cell activation. J Immunol 146: 4092-4098, 1991.

42. Möller P, Henne C, Leithäuser F, et al: Coregulation of the APO-1 antigen with intercellular adhesion molecule-1 (CD54) in tonsillar B cells and coordinate expression in follicular center $\mathrm{B}$ cells and in follicle center and mediastinal B-cell lymphomas. Blood 81: 2067-2075, 1993

43. Grobholz R, Zentgraf H, Köhrmann KU and Bleyl U: Bax, Bcl-2, fas and Fas-L antigen expression in human seminoma: correlation with the apoptotic index. APMIS 110: 724-732, 2002

44. Jha P, Sohn JH, Xu Q, Wang Y, Kaplan HJ, Bora PS and Bora NS: Suppression of complement regulatory proteins (CRPs) exacerbates experimental autoimmune anterior uveitis (EAAU). J Immunol 176: 7221-7231, 2006.

45. Donev RM, Gray LC, Sivasankar B, Hughes TR, van den Berg CW and Morgan BP: Modulation of CD59 expression by restrictive silencer factor-derived peptides in cancer immunotherapy for neuroblastoma. Cancer Res 68: 5979-5987, 2008. 